\title{
An energy-based limit state function for estimation of structural reliability in shock environments
}

\author{
Michael A. Guthrie \\ Analytical Structural Dynamics, Org. 1523, Sandia National Laboratories, MS 0346, P.O. Box 5800, Albuquerque, \\ NM 87185, USA \\ Tel.: +1 505284 1357; E-mail: maguthr@sandia.gov
}

Received 6 September 2012

Revised 17 December 2012

Accepted 13 April 2013

\begin{abstract}
A limit state function is developed for the estimation of structural reliability in shock environments. This limit state function uses peak modal strain energies to characterize environmental severity and modal strain energies at failure to characterize the structural capacity. The Hasofer-Lind reliability index is briefly reviewed and its computation for the energy-based limit state function is discussed. Applications to two degree of freedom mass-spring systems and to a simple finite element model are considered. For these examples, computation of the reliability index requires little effort beyond a modal analysis, but still accounts for relevant uncertainties in both the structure and environment. For both examples, the reliability index is observed to agree well with the results of Monte Carlo analysis. In situations where fast, qualitative comparison of several candidate designs is required, the reliability index based on the proposed limit state function provides an attractive metric which can be used to compare and control reliability.
\end{abstract}

Keywords: Shock, structural reliability, limit state function

\section{Introduction}

A great deal of literature, beginning with the work of Hudson [1] and Housner [2,3], has focused on the use of energy to characterize earthquake excitation of structures. These energy metrics have become widely accepted in the earthquake engineering community, but until recently have received little attention elsewhere. However, recent work in the structural dynamics field has focused on using energy to characterize the severity of shock and vibration environments [4-6]. These energy-based metrics offer a number of advantages over the more traditional shock response spectrum, such as greater meaning for multiple degree of freedom systems, closer correspondence to material failure criteria, and greater ability to distinguish between waveforms with different temporal characteristics and durations. Perhaps the greatest benefit is that energy-based characterization of shock and vibration environments allows for the comparison of component test environments with requirements through scalar estimates of margin, which can then be used in reliability analysis.

A natural extension of the energy-based characterization of shock and vibration environments is the prediction of component reliability in these environments using energy-based severity metrics. In this context, component margin testing is replaced by finite element modeling in order to establish the energy capacity of the structure. The energy capacity can then be compared probabilistically with the energy demands imposed by the environment to obtain estimates of component reliability using methods from the structural reliability literature (e.g., $[7,8])$. 
An important concept from the structural reliability literature is that of the limit state function. Limit state functions are scalar functions $g(Z)$ such that failure occurs if and only if $g(Z)<0$, where the variables $Z$ are called the basic variables. The hypersurface defined by $g(Z)=0$ is known as the limit state surface and it partitions the space of basic variables into a safe set for which $g(Z)>0$ and a failure set for which $g(Z)<0$. Limit state functions are used throughout the structural reliability literature to define failure in terms of applied forces, yield stresses, dimensions, etc.

Structural reliability methods are often classified by the amount of statistical information about the basic variables which they require [7]. In level II methods, it is assumed that estimates of the expected value and covariance of the basic variables are available. The advantage of these methods is that they eliminate the need for determining probability distributions for each basic variable. The cost of this simplification is that level II methods cannot predict reliability directly; instead they provide reliability indices which can be used to compare and control reliability. For example, these methods could be very useful during the design process when one wishes to determine which of several designs is more reliable in a given environment or to assess the influence of a design change on the reliability. However, the reliability index does not have a direct interpretation in terms of the probability of failure without assuming probability distributions for the basic variables - its value is as a predictor of reliability for one structure relative to another. Perhaps the most common level II method is the use of the Hasofer-Lind reliability index [9], which is defined as the shortest distance between the mean point and the limit state surface in the space of standardized, decorrelated basic variables. In contrast to level II methods, level III methods predict reliability but require probability density functions for each of the basic variables.

In this paper, a limit state function based upon the peak modal strain energies and modal strain energies at failure is developed for the estimation of structural reliability in shock environments. Due to the difficulty of approximating the probability distributions for these basic variables, level II reliability methods are considered exclusively. The Hasofer-Lind reliability index can be calculated based upon the proposed limit state function and provides a measure of the structure's reliability. This approach could be very valuable during the design process, when fast, qualitative comparison of the relative reliabilities of various design options is required. In such situations, Monte Carlo analysis is often too computationally intensive to provide the fast turnaround required to support design studies. The reliability approach developed here requires little effort beyond a modal analysis, but still accounts for relevant uncertainties in both the structure and environment. A second advantage of this approach is that it provides important information about the sensitivity of the structure's reliability to the frequency content of the input.

This document begins with the mathematical development of the limit state function, followed by a brief description of the Hasofer-Lind reliability index and its computation for the proposed limit state function. The reliability approach is then applied to two degree of freedom (DOF) base excited mass-spring systems as well as a simple finite element model. In both cases, the reliability indices are compared with the results of Monte Carlo analysis and it is seen that the two approaches produce similar results and that the reliability approach is much faster and easier to implement.

\section{Development of limit state function}

Consider a general linear structure with mass-normalized mode shapes $\phi_{i}$ and natural frequencies $\omega_{i}$ which can be accurately represented in terms of a subset of $n$ of these modes. Suppose that the structure is subjected to a shock input through its base which is characterized in terms of peak modal strain energies $E_{i}$ and that failure of the structure is defined through $m$ inequalities of the form:

$$
\left|f_{j}(d)\right|>f_{\text {fail }, j} \text { for any } j=1 \ldots m
$$

where $f_{j}(d)$ can denote any linear function of displacement used to define failure of the structure (e.g., force, stress, etc.) and $f_{f a i l, j} \geqslant 0$ denotes the value of that quantity at which failure occurs. Both the shock input and the structure are treated probabilistically and a measure of the reliability is desired. For this problem, a limit state function can be developed for each of the $m$ constraints. Begin by noting that for a general shape $\Phi \alpha$ where $\alpha$ is an arbitrary vector of modal coordinates and $\Phi$ denotes the mass-normalized modal matrix, the strain energy in mode $i$ is:

$$
\varepsilon_{i}=\frac{1}{2} \omega_{i}^{2} \alpha_{i}^{2} \text { for } i=1 \ldots n
$$


Next, consider scaling the shape $\Phi \alpha$ by a constant $c$ such that $f_{j}(d)=f_{\text {fail }, j}$. Since $f_{j}(d)$ is linear,

$$
c=\frac{f_{\text {fail }, j}}{f_{j}(\Phi \alpha)}
$$

The strain energy in mode $i$ when the structure is scaled such that $f_{j}(d)=f_{\text {fail }, j}$ is therefore:

$$
\varepsilon_{i}=\frac{1}{2} \omega_{i}^{2} \alpha_{i}^{2} \frac{f_{\text {fail }, j}^{2}}{f_{j}(\Phi \alpha)^{2}} \text { for } i=1 \ldots n, j=1 \ldots m
$$

By rewriting the modal coordinates as

$$
\alpha=\left[\begin{array}{c}
\alpha_{1} \\
\vdots \\
\alpha_{n}
\end{array}\right]=\alpha_{n}\left[\begin{array}{c}
\alpha_{1} / \alpha_{n} \\
\vdots \\
\alpha_{n-1} / \alpha_{n} \\
1
\end{array}\right] \stackrel{\text { def }}{=} \alpha_{n}\left[\begin{array}{c}
\xi_{1} \\
\vdots \\
\xi_{n-1} \\
1
\end{array}\right] \stackrel{\text { def }}{=} \alpha_{n} \xi
$$

Equation (2) can be rewritten as

$$
\begin{aligned}
\varepsilon_{i} & =\frac{1}{2} \omega_{i}^{2} \xi_{i}^{2} \frac{f_{\text {fail }, j}^{2}}{f_{j}(\Phi \xi)^{2}} \text { for } i=1 \ldots n-1, j=1 \ldots m \\
\varepsilon_{n} & =\frac{1}{2} \omega_{n}^{2} \frac{f_{\text {fail }, j}^{2}}{f_{j}(\Phi \xi)^{2}} \text { for } j=1 \ldots m
\end{aligned}
$$

For each value of $j$, these equations define an $n-1$ dimensional strain energy at failure surface parameterized by $\xi_{1} \ldots \xi_{n-1}$. A more useful definition of these surfaces than the parametric one given in Eqs (6) and (7) would relate the modal strain energies $\varepsilon_{i}$ to one another. Therefore the following mathematical form is proposed to describe the strain energy at failure surfaces:

$$
\varepsilon_{n}=\left.\varepsilon_{\text {fail }, n}\right|_{j}\left(1+\sum_{i=1}^{n-1} s_{i} \sqrt{\frac{\varepsilon_{i}}{\left.\varepsilon_{\text {fail }, i}\right|_{j}}}\right)^{2} \text { for } j=1 \ldots m
$$

where $s_{i}= \pm 1 \forall i$ and the strain energy at failure of element $j$ for pure deformation in mode $i$ is

$$
\left.\varepsilon_{\text {fail }, i}\right|_{j} \stackrel{\text { def }}{=} \frac{1}{2} \omega_{i}^{2} \frac{f_{\text {fail }, j}^{2}}{f_{j}\left(\phi_{i}\right)^{2}}
$$

In order to verify that Eq. (8) represents the surfaces defined parametrically in Eqs (6) and (7), substitute Eqs (6), (7), and (9) into Eq. (8) and simplify to obtain:

$$
f_{j}\left(\phi_{n}\right)^{2}=\left(\left|f_{j}(\Phi \xi)\right|+\sum_{i=1}^{n-1} s_{i}\left|\xi_{i}\right|\left|f_{j}\left(\phi_{i}\right)\right|\right)^{2}
$$

Since the functions $f_{j}(x)$ are linear, this can be rewritten as

$$
\begin{aligned}
f_{j}\left(\phi_{n}\right)^{2} & =\left(\left|\sum_{i=1}^{n} \xi_{i} f_{j}\left(\phi_{i}\right)\right|+\sum_{i=1}^{n-1} s_{i}\left|\xi_{i}\right|\left|f_{j}\left(\phi_{i}\right)\right|\right)^{2} \\
& =\left(\operatorname{sgn}\left(\sum_{i=1}^{n} \xi_{i} f_{j}\left(\phi_{i}\right)\right) \sum_{i=1}^{n} \xi_{i} f_{j}\left(\phi_{i}\right)+\sum_{i=1}^{n-1} \operatorname{sgn}\left(\xi_{i}\right) \operatorname{sgn}\left(f_{j}\left(\phi_{i}\right)\right) s_{i} \xi_{i} f_{j}\left(\phi_{i}\right)\right)
\end{aligned}
$$


By choosing

$$
s_{i}=-\operatorname{sgn}\left(\xi_{i}\right) \operatorname{sgn}\left(\sum_{k=1}^{n} \xi_{k} f_{j}\left(\phi_{k}\right)\right) \operatorname{sgn}\left(f_{j}\left(\phi_{i}\right)\right)
$$

the equation above becomes

$$
f_{j}\left(\phi_{n}\right)^{2}=\left(\operatorname{sgn}\left(\sum_{i=1}^{n} \xi_{i} f_{j}\left(\phi_{i}\right)\right)\left(\sum_{i=1}^{n} \xi_{i} f_{j}\left(\phi_{i}\right)-\sum_{i=1}^{n-1} \xi_{i} f_{j}\left(\phi_{i}\right)\right)\right)^{2}
$$

which is clearly true. Thus the functional form defined in Eq. (8) is valid as long as the functions $f_{j}(x)$ are linear. Equation (8) defines $2^{n-1}$ strain energy at failure surfaces associated with each of the permutations of the $s_{i}$ for each of the $m$ constraints, but if $\varepsilon_{1} \ldots \varepsilon_{n-1}$ are small enough that

$$
\sum_{i=1}^{n-1} \sqrt{\frac{\varepsilon_{i}}{\left.\varepsilon_{\text {fail }, i}\right|_{j}}}<1
$$

then the surface with $s_{i}=-1 \forall i$ is the most conservative in the sense that the value of $\varepsilon_{n}$ required for failure is less for $s_{i}=-1 \forall i$ than for any other permutation of the $s_{i}$. If the condition in Eq. (14) does not hold, failure of the structure is possible since one or more of the limit state surfaces as defined by Eq. (8) has been exceeded. Therefore it is assumed that failure occurs whenever Eq. (14) does not hold, and that failure occurs on the branch of the strain energy at failure surface with $s_{i}=-1 \forall i$ when the condition in Eq. (14) does hold. These assumptions lead to the following limit state function for each constraint in the $2 n$ basic variables $E_{i}$ and $\left.\varepsilon_{f a i l, i}\right|_{j}$ :

$$
g_{j}\left(E_{i},\left.\varepsilon_{\text {fail }, i}\right|_{j}\right)= \begin{cases}\left.\varepsilon_{\text {fail }, n}\right|_{j}\left(1-\sum_{i=1}^{n-1} \sqrt{\frac{E_{i}}{\left.\varepsilon_{\text {fail }, i}\right|_{j}}}\right)^{2}-E_{n} & \text { if } \sum_{i=1}^{n-1} \sqrt{\frac{E_{i}}{\left.\varepsilon_{\text {fail }, i}\right|_{j}}}<1 \\ \left.\varepsilon_{\text {fail }, n}\right|_{j}\left(1-\sum_{i=1}^{n-1} \sqrt{\frac{E_{i}}{\left.\varepsilon_{\text {fail }, i}\right|_{j}}}\right)-E_{n} \quad \text { otherwise }\end{cases}
$$

Note that the second part of this definition is arbitrary: any limit state function which is strictly negative when the condition in Eq. (14) does not hold would be valid since it is assumed that the structure fails in this case. The definition in Eq. (15) has been adopted for computational convenience. It should be noted that the approach developed here is limited to structures for which failure can be defined in terms of mechanical criteria of the type specified in Eq. (1). Since it depends upon the FEM to establish the strain energy at failure in each of the structure's modes, the approach is unable to predict failures which cannot be modeled using the underlying FEM (e.g., electrical failures). While the derivation above is only valid for failure criteria which depend linearly on displacement, the final example problem considered in this paper suggests that the limit state function can be applied when the failure criterion does not depend linearly on displacement without great loss of accuracy. The extension of the limit state function to these cases will be considered more rigorously in future work.

Two important conservative assumptions have been made in developing the limit state function of Eq. (15) from Eq. (8). First, each of the modal strain energies has been assumed to equal its peak value. In reality, the modal strain energies $\varepsilon_{i}$ are functions of time which each peak at distinct times, but by setting these energies equal to their peak values $E_{i}$, it is assumed that they all peak simultaneously. The second conservative assumption made in developing Eq. (15) is in using the most conservative branch of the strain energy at failure surface from Eq. (8). Thus, if $g_{j}>0 \forall j$, failure as defined by Eq. (1) is impossible, but if $g_{j}<0$ for one or more values of $j$ the system does not necessarily fail since it might fail on one of the less conservative branches of Eq. (8). The physical meaning of these branches is explored in an example problem later in this paper. It is anticipated that the assumptions underlying the limit state function will introduce considerable conservative bias to estimates of the probability of failure if the limit state function is used for level III reliability analysis. However, it is also anticipated that these assumptions will be consistently conservative from structure to structure so that, when the limit state function is used for level II reliability analysis, the reliability indices will accurately reflect the reliability of one structure relative to another. The reliability indices will thus still be useful for their intended purpose: to compare and control the reliability of structures. 


\section{Hasofer-Lind reliability index}

One of the most widely used level II reliability methods is the Hasofer-Lind reliability index [9]. This index represents the minimum distance between the origin and the limit state surface in the space of standardized, decorrelated basic variables. For a wide variety of distributions, the probability content of the basic variables will decline exponentially with distance from the origin, so the probability of failure will often be dominated by the small region of the failure domain nearest the origin. In these cases, the distance of this region from the origin, or the Hasofer-Lind reliability index, provides a good measure of reliability. For a problem with only a single basic variable, this index is equal to the distance from the mean point to the failure point measured in standard deviations of the basic variable, which corresponds to the Cornell reliability index [10]. The mathematics of the Hasofer-Lind reliability index are briefly reviewed below and applied to the limit state function defined in Eq. (15).

Denote the vector of basic variables by $Z$. For the problem considered in this paper,

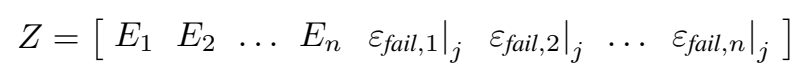

The limit state function defined in Eq. (15) can be rewritten in terms of the basic variables as

$$
g_{j}(Z)= \begin{cases}Z_{2 n}\left(1-\sum_{i=1}^{n-1} \sqrt{\frac{Z_{i}}{Z_{i+n}}}\right)^{2}-Z_{n} \quad \text { if } \sum_{i=1}^{n-1} \sqrt{\frac{Z_{i}}{Z_{i+n}}}<1 \\ Z_{2 n}\left(1-\sum_{i=1}^{n-1} \sqrt{\frac{Z_{i}}{Z_{i+n}}}\right)-Z_{n} \quad \text { otherwise }\end{cases}
$$

In general, the basic variables are correlated and have non-zero means and non-unit variances. Therefore the covariance matrix $C_{Z}$ will be non-diagonal and the expected value $\mathbf{E}[Z] \neq 0$. Define the standardized, decorrelated variables $X$ as follows:

$$
X=A(Z-\boldsymbol{E}[Z])
$$

where the matrix $A$ is chosen such that $X$ has covariance equal to the identity matrix:

$$
C_{X}=A C_{Z} A^{T}=I
$$

The matrix $A$ satisfying Eq. (19) has rows which are the transposed eigenvectors of $C_{Z}, v_{i}^{T}$, divided by the square root of the corresponding eigenvalue, $r_{i}$ :

$$
A=\left[\begin{array}{c}
v_{1}^{T} / \sqrt{r_{1}} \\
\vdots \\
v_{2 n}^{T} / \sqrt{r_{2 n}}
\end{array}\right]
$$

The variables $X$ defined in this way are uncorrelated and have zero mean and unit variance. The Hasofer-Lind reliability index is defined as the minimum distance from the origin to the limit state surface in $X$-space:

$$
\beta_{j}=\min _{g_{j}(x)=0} \sqrt{x^{T} x}
$$

where $x$ denotes a realization of the standardized variables $X$. Equation (21) can be restated in terms of realizations of the basic variables $Z$ using Eqs (18) and (19):

$$
\beta_{j}=\min _{g_{j}(z)=0} \sqrt{(z-\boldsymbol{E}[Z])^{T} C_{Z}^{-1}(z-\boldsymbol{E}[Z])}
$$


The point $z^{*}$ satisfying Eq. (22) is known as the most probable point (MPP), since it is the point in the failure domain with the greatest probability content. In [9], Hasofer and Lind proposed a simple iterative scheme for finding the MPP:

$$
z^{(k+1)}=\boldsymbol{E}[Z]+C_{Z} \nabla g_{j}\left(z^{(k)}\right) \frac{\left(z^{(k)}-\boldsymbol{E}[Z]\right)^{T} \nabla g_{j}\left(z^{(k)}\right)-g_{j}\left(z^{(k)}\right)}{\nabla g_{j}\left(z^{(k)}\right)^{T} C_{Z} \nabla g_{j}\left(z^{(k)}\right)}
$$

where the initial point is taken to be the mean $\left(z^{(0)}=\boldsymbol{E}[Z]\right)$ and the gradient of the limit state function is

$$
\begin{aligned}
& \left(\nabla g_{j}\right)_{i}(Z)=\left\{\begin{array}{ll}
-Z_{2 n}\left(1-\sum_{k=1}^{n-1} \sqrt{\frac{Z_{k}}{Z_{k+n}}}\right) \frac{1}{\sqrt{Z_{i} Z_{i+n}}} & \text { if } \sum_{k=1}^{n-1} \sqrt{\frac{Z_{k}}{Z_{k+n}}}<1 \\
-\frac{1}{2} Z_{2 n} \frac{1}{\sqrt{Z_{i} Z_{i+n}}} & \text { otherwise }
\end{array} \text { for } i=1 \ldots n-1\right. \\
& \left(\nabla g_{j}\right)_{n}(Z)=-1 \\
& \left(\nabla g_{j}\right)_{i}(Z)=\left\{\begin{array}{lll}
Z_{2 n}\left(1-\sum_{k=1}^{n-1} \sqrt{\frac{Z_{k}}{Z_{k+n}}}\right) \sqrt{\frac{Z_{i-n}}{Z_{i}^{3}}} & \text { if } \sum_{k=1}^{n-1} \sqrt{\frac{Z_{k}}{Z_{k+n}}}<1 & \text { for } i=n+1 \ldots 2 n-1 \\
\frac{1}{2} Z_{2 n} \sqrt{\frac{Z_{i-n}}{Z_{i}^{3}}} & \text { otherwise }
\end{array}\right. \\
& \left(\nabla g_{j}\right)_{2 n}(Z)= \begin{cases}\left(1-\sum_{k=1}^{n-1} \sqrt{\frac{Z_{k}}{Z_{k+n}}}\right)^{2} & \text { if } \sum_{k=1}^{n-1} \sqrt{\frac{Z_{k}}{Z_{k+n}}}<1 \\
\left(1-\sum_{k=1}^{n-1} \sqrt{\frac{Z_{k}}{Z_{k+n}}}\right) & \text { otherwise }\end{cases}
\end{aligned}
$$

Since Eq. (15) defines $m$ limit state functions, Eq. (23) must be applied with each of these to find $m$ independent reliability indices $\beta_{1}, \beta_{2}, \ldots \beta_{m}$. The system reliability index is then equal to the minimum of these:

$$
\beta=\min _{j=1 \ldots m} \beta_{j}
$$

The vector $x^{*}$ satisfying Eq. (21) is related to the sensitivity of the reliability index to the decorrelated, standardized variables $x_{i}$ :

$$
x_{i}^{*}=\beta \frac{\partial \beta}{\partial x_{i}}
$$

The change in the reliability index for a change in the expected value of each of the original basic variables $Z_{i}$ by one standard deviation can be found by combining Eqs (18) and (26):

$$
\frac{\partial \beta}{\partial\left(\boldsymbol{E}\left[Z_{i}\right] / \sigma_{i}\right)}=-\frac{\sigma_{i}}{\beta} \sum_{j=1}^{2 n} A_{j i} x_{j}^{*}
$$

In order to determine the Hasofer-Lind reliability index, estimates of the means, variances, and correlation coefficients for the peak modal strain energies and modal strain energies at failure are required. It is assumed here that the mean and variance for each of these variables is available and that the peak modal strain energies are uncorrelated with the modal strain energies at failure. Since the peak modal strain energies are largely functions of the environment and the modal strain energies at failure are functions of the structural properties, it is expected that this will typically be an accurate assumption. The correlation between the peak modal strain energies is a function of the environment, and an analysis of this correlation for general environments is beyond the scope of this paper. For 

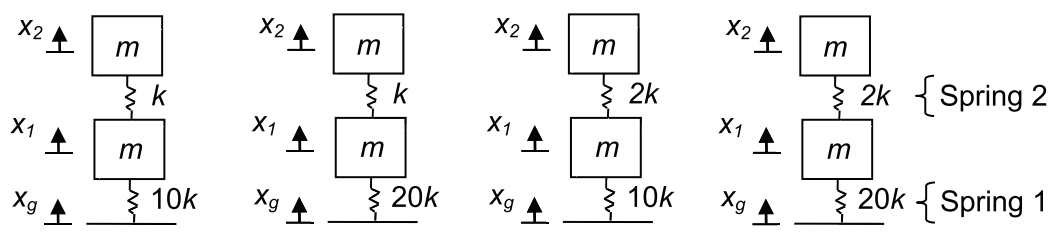

Fig. 1. Base-excited, two DOF systems (from left to right, structures 1-4).

well-separated natural frequencies and environments with broad frequency content, it is expected that this correlation will often be small, so the peak modal strain energies are assumed to be uncorrelated with one another in the example problems of Sections 4 and 5. Furthermore, the modal strain energies at failure are assumed to be perfectly correlated with one another in these problems. This assumption holds exactly for cases in which the natural modes and frequencies are deterministic and only the failure criteria are uncertain, and it is expected that it will remain an accurate assumption for cases in which the variability in the natural modes and frequencies is small compared to the variability in the failure criteria. Nonetheless, the approach is general and can account for arbitrary correlations amongst the basic variables when these correlations are known.

\section{Application to mass-spring systems}

To illustrate the use of the energy-based limit state function in a level II reliability analysis, the four base-excited, two DOF systems shown in Fig. 1 are considered. While it is not clear a priori which of these systems is most reliable in a given shock environment, this section will demonstrate that the Hasofer-Lind reliability index calculated using the energy-based limit state function provides an excellent metric of each system's reliability. Each system is damped with 5\% modal damping and fails when the force in either of the springs exceeds the corresponding failure force $f_{f a i l, 1}$ or $f_{f a i l, 2}$. The strain energy at failure of spring $j$ for pure deformation in mode $i$ is given by:

$$
\left.\varepsilon_{\text {fail }, i}\right|_{j}=\frac{1}{2} \omega_{i}^{2} \frac{f_{\text {fail }, j}^{2}}{\left|P_{j} \phi_{i}\right|^{2}}
$$

where $P$ denotes the matrix that, when post-multiplied by the displacement, produces the spring forces:

$$
P=\left[\begin{array}{cc}
k_{1} & 0 \\
-k_{2} & k_{2}
\end{array}\right]
$$

and $P_{j}$ denotes row $j$ of this matrix. Consider the following nominal values of the system parameters:

$$
\bar{m}=0.01 \mathrm{~kg}, \bar{k}=400 \pi^{2} \mathrm{~N} / \mathrm{m}, \bar{f}_{\text {fail }, 1}=10 \mathrm{~N}, \text { and } \bar{f}_{\text {fail }, 2}=3.9 \mathrm{~N}
$$

Each system is subjected to the nominal base acceleration shown in Fig. 2. The nominal natural frequencies, effective modal masses, strain energies at failure, and peak modal strain energies for each system are given in Table 1 . The peak modal strain energies are calculated by integrating the modal responses to the given base acceleration using the central difference method and then employing the following equation:

$$
E_{i}=\frac{1}{2} \omega_{i}^{2} \alpha_{i, \max }^{2}
$$

where $\alpha_{i, \max }$ denotes the maximum of the absolute value of the modal displacement in mode $i$.

The basic variables for spring $j$ are:

$$
Z=\left[\begin{array}{llll}
Z_{1} & Z_{2} & Z_{3} & Z_{4}
\end{array}\right]=\left[\begin{array}{llll}
E_{1} & E_{2} & \left.\varepsilon_{f a i l, 1}\right|_{j} & \left.\varepsilon_{f a i l, 2}\right|_{j}
\end{array}\right]
$$

For each structure, the expected values of the basic variables are set equal to the nominal values from Table 1. In addition, each basic variable is assigned a coefficient of variation equal to 0.2. The correlation between basic 
Table 1

Natural frequencies, effective modal masses, strain energies at failure, and peak modal strain energies for two DOF structures

\begin{tabular}{|c|c|c|c|c|}
\hline Structure & 1 & 2 & 3 & 4 \\
\hline First natural frequency, $\omega_{1}(\mathrm{~Hz})$ & 94.9 & 97.5 & 127.1 & 134.2 \\
\hline Second natural frequency, $\omega_{2}(\mathrm{~Hz})$ & 333.2 & 458.8 & 351.9 & 471.1 \\
\hline Modal participation factor - mode 1 & -0.773 & -0.742 & -0.828 & -0.773 \\
\hline Modal participation factor - mode 2 & -0.634 & -0.671 & -0.561 & -0.634 \\
\hline Peak strain energy in mode $1, E_{1}(\mathrm{~N} \cdot \mathrm{m})$ & $7.89 \times 10^{-4}$ & $7.04 \times 10^{-4}$ & $5.66 \times 10^{-4}$ & $5.14 \times 10^{-4}$ \\
\hline Peak strain energy in mode $2, E_{2}(\mathrm{~N} \cdot \mathrm{m})$ & $2.31 \times 10^{-4}$ & $2.78 \times 10^{-4}$ & $1.61 \times 10^{-4}$ & $2.67 \times 10^{-4}$ \\
\hline Strain energy in mode 1 at failure of spring $1,\left.\varepsilon_{\text {fail, } 1}\right|_{1}(\mathrm{~N} \cdot \mathrm{m})$ & $1.18 \times 10^{-2}$ & $1.21 \times 10^{-2}$ & $5.72 \times 10^{-3}$ & $5.88 \times 10^{-3}$ \\
\hline Strain energy in mode 2 at failure of spring $1,\left.\varepsilon_{\text {fail }, 2}\right|_{1}(\mathrm{~N} \cdot \mathrm{m})$ & $1.40 \times 10^{-3}$ & $6.68 \times 10^{-4}$ & $1.63 \times 10^{-3}$ & $7.10 \times 10^{-4}$ \\
\hline Strain energy in mode 1 at failure of spring $2, \varepsilon_{\text {fail }, 1}{ }_{2}(\mathrm{~N} \cdot \mathrm{m})$ & $2.20 \times 10^{-3}$ & $2.03 \times 10^{-3}$ & $1.24 \times 10^{-3}$ & $1.08 \times 10^{-3}$ \\
\hline Strain energy in mode 2 at failure of spring $2, \varepsilon_{f a i l 2}$ (N.m) & $1.79 \times 10^{-2}$ & $3.69 \times 10^{-2}$ & $4.35 \times 10^{-3}$ & $8.94 \times 10^{-3}$ \\
\hline
\end{tabular}

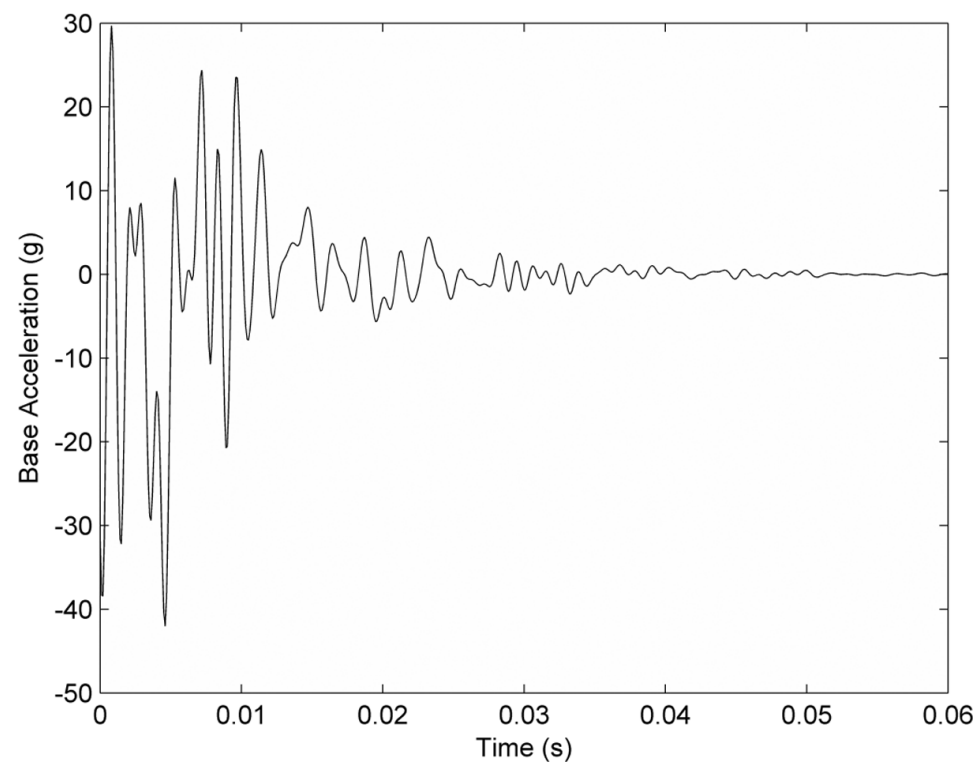

Fig. 2. Nominal base acceleration applied to two DOF system.

variables is treated as discussed previously: i.e., the peak modal strain energies are uncorrelated with the modal strain energies at failure, the modal strain energies at failure are perfectly correlated with one another, and the peak modal strain energies are uncorrelated with one another. Given these assumptions, the covariance matrix for $Z$ takes the form

$$
C_{Z}=\left[\begin{array}{cccc}
0.04 \boldsymbol{E}\left[Z_{1}\right]^{2} & 0 & 0 & 0 \\
& 0.04 \boldsymbol{E}\left[Z_{2}\right]^{2} & 0 & 0 \\
& & 0.04 \boldsymbol{E}\left[Z_{3}\right]^{2} & 0.04 \boldsymbol{E}\left[Z_{3}\right] \boldsymbol{E}\left[Z_{4}\right] \\
\text { sym } & & & 0.04 \boldsymbol{E}\left[Z_{4}\right]^{2}
\end{array}\right]
$$

For these two DOF structures, the limit state function defined in Eq. (17) simplifies to

$$
g(Z)= \begin{cases}Z_{4}\left(1-\sqrt{\frac{Z_{1}}{Z_{3}}}\right)^{2}-Z_{2} & \text { if } Z_{1}<Z_{3} \\ Z_{4}\left(1-\sqrt{\frac{Z_{1}}{Z_{3}}}\right)-Z_{2} & \text { otherwise }\end{cases}
$$


Table 2

Reliability indices, sensitivity vectors, and Monte Carlo reliabilities for two DOF structures

\begin{tabular}{lcccc}
\hline Structure & 1 & 2 & 3 & 4 \\
\hline Hasofer-lind reliability index, $\beta$ & 2.20 & 0.96 & 1.02 & 0.78 \\
Spring governing $\beta$ & Spring 2 & Spring 1 & Spring 2 & Spring 1 \\
Sensitivity vector from Eq. (20) & -0.395 & -0.186 & -0.502 & -0.232 \\
& -0.079 & -0.480 & -0.146 & -0.466 \\
& 0.779 & 0.230 & 0.670 & 0.278 \\
& 0.137 & 0.627 & 0.182 & 0.575 \\
Overall reliability from Monte Carlo & $98.85 \%$ & $91.47 \%$ & $93.23 \%$ & $84.41 \%$ \\
Spring 1 reliability - Monte Carlo & $99.79 \%$ & $92.11 \%$ & $99.82 \%$ & $91.96 \%$ \\
Spring 2 reliability - Monte Carlo & $99.06 \%$ & $99.36 \%$ & $93.41 \%$ & $92.45 \%$ \\
\hline
\end{tabular}

which has gradient from Eq. (24):

$$
\nabla g=\left[\begin{array}{c}
-Z_{4}\left(1-\sqrt{\frac{Z_{1}}{Z_{3}}}\right) \frac{1}{\sqrt{Z_{1} Z_{3}}} \\
-1 \\
Z_{4}\left(1-\sqrt{\frac{Z_{1}}{Z_{3}}}\right) \sqrt{\frac{Z_{1}}{Z_{3}^{3}}} \\
\left(1-\sqrt{\frac{Z_{1}}{Z_{3}}}\right)^{2}
\end{array}\right] \text { if } Z_{1}<Z_{3} \text { and } \nabla g=\left[\begin{array}{c}
-\frac{1}{2} Z_{4} \frac{1}{\sqrt{Z_{1} Z_{3}}} \\
-1 \\
\frac{1}{2} Z_{4} \sqrt{\frac{Z_{1}}{Z_{3}^{3}}} \\
\left(1-\sqrt{\frac{Z_{1}}{Z_{3}}}\right)
\end{array}\right] \text { otherwise }
$$

Given these expressions for the limit state function and its gradient as well as the expected value and covariance of the basic variables, the iterative procedure given in Eq. (23) can be applied to find the MPP and the Hasofer-Lind reliability index for each of the structures considered. The reliability index, the spring with the minimum reliability index, and the sensitivity vector from Eq. (27) for each structure are stated in Table 2. The reliability indices suggest that structure 1 is by far the most reliable in the given environment, followed by structures 3,2 , and 4 . The results also suggest that structures 1 and 3 are most likely to fail at spring 2, while structures 2 and 4 are most likely to fail at spring 1 , since these are the springs governing the reliability index for each structure. The sensitivity vectors listed in Table 2 provide information that could be useful in the design process. For example, the reliability of structure 1 is most sensitive to the peak strain energy in mode 1 and strain energy at failure in mode 1 . This suggests design changes that could make structure 1 more reliable, such as increasing the damping in mode 1 , shifting the frequency of mode 1 so it receives less energy from the input, and increasing the failure force for spring 2 . While much of this information could be easily determined through other means for these simple systems, the technique presented here can be readily applied to more complicated structures, as illustrated in Section 5.

In order to provide confidence in the results from the reliability analysis, a Monte Carlo analysis was also performed. Base accelerations were generated as exponentially decayed sums of sines at each of the structure's natural frequencies:

$$
A_{g}=e^{-c t} \sum_{i=1}^{2} A_{i} \sin \omega_{i} t
$$

where the decay constant is chosen to be $c=151 / \mathrm{s}$. The coefficients $A_{i}$ are independent and normally distributed with means and variances selected such that the base acceleration delivers peak modal strain energies which have means and standard deviations matching the nominal values listed in Table 1 and the assumed coefficient of variation of 0.2. That is, the means and variances of the coefficients $A_{i}$ were selected to satisfy the following system of equations:

$$
H(y) \equiv h(y)-h^{*}=0
$$

where $y$ is a vector containing the means and variances of the coefficients $A_{i}, h(y)$ denotes a vector of the means and standard deviations of the peak modal strain energies for a given value of $y$, and $h^{*}$ represents a vector of the 
Table 3

Parameters for generation of base accelerations used in Monte Carlo analysis of two DOF structures

\begin{tabular}{|c|c|c|c|c|c|c|c|c|}
\hline \multirow[b]{2}{*}{ Mode \# } & \multicolumn{2}{|c|}{ Structure 1} & \multicolumn{2}{|c|}{ Structure 2} & \multicolumn{2}{|c|}{ Structure 3} & \multicolumn{2}{|c|}{ Structure 4} \\
\hline & $\mu(\mathrm{g})$ & $\sigma^{2}\left(g^{2}\right)$ & $\mu(\mathrm{g})$ & $\sigma^{2}\left(g^{2}\right)$ & $\mu(\mathrm{g})$ & $\sigma^{2}\left(g^{2}\right)$ & $\mu(\mathrm{g})$ & $\sigma^{2}\left(g^{2}\right)$ \\
\hline 1 & 4.33 & 0.200 & 4.45 & 0.199 & 4.23 & 0.180 & 4.37 & 0.205 \\
\hline 2 & 6.60 & 0.499 & 9.10 & 0.950 & 6.55 & 0.490 & 9.50 & 0.970 \\
\hline
\end{tabular}

desired means and standard deviations of the peak modal strain energies. This equation can be solved numerically using Newton's method:

$$
y^{\ell+1}=y^{\ell}-\left[J\left(y^{\ell}\right)\right]^{-1} H\left(y^{\ell}\right) \text { where }\left[J\left(y^{\ell}\right)\right]_{i j}=\frac{\partial H_{i}}{\partial y_{j}}\left(y=y^{\ell}\right)
$$

in which $y^{\ell}$ denotes the value of $y$ on iteration $\ell$. The Jacobian $J$ is evaluated numerically on each iteration by perturbing each of the variables in $y$ in isolation:

$$
\left[J\left(y^{\ell}\right)\right]_{i j} \approx \frac{H_{i}\left(\tilde{y}^{\ell}\right)-H_{i}\left(y^{\ell}\right)}{\delta y_{j}^{\ell}} \text { where } \tilde{y}_{q}^{\ell}= \begin{cases}y_{q}^{\ell} & \text { for } q \neq j \\ (1+\delta) y_{j}^{\ell} & \text { for } q=j\end{cases}
$$

A value of $\delta=10^{-1}$ was used in this work. To evaluate the function $H(y)$ in Eqs (38) and (39), Monte Carlo analysis was performed in which $10^{4}$ realizations of the base acceleration were formed using Eq. (36) with the current value of $y$, the modal responses were integrated for each of these inputs using the central difference method, and the peak modal strain energies were calculated using Eq. (31). This leads to estimates of the means and standard deviations of the peak modal strain energies for a given value of $y$, i.e. $h(y)$. For this problem, the iterations in Eq. (38) begin with an initial guess of $y^{0}=\left[10 \mathrm{~g}, 10 \mathrm{~g}, 1 \mathrm{~g}^{2}, 1 \mathrm{~g}^{2}\right]$ and iterations continue until each entry of $H(y)$ is less than or equal to $1 \%$ of the corresponding entry of $h^{*}$. In order to ensure that the variances obtained in this way are positive, on each iteration the update in Eq. (38) is repeatedly multiplied by 0.5 until $\left[y_{n+1}^{\ell+1} \ldots y_{2 n}^{\ell+1}\right]>0$. The means and variances determined in this manner for the two DOF structures of Fig. 1 are given in Table 3. By synthesizing the base accelerations as described here, a family of time domain inputs is created which has the same second-moment properties as assumed for the input in the reliability analysis.

In addition to generating random base accelerations, random failure forces for the two springs must also be chosen. On each Monte Carlo trial, strain energies at failure of each the springs in the first mode are drawn from Weibull distributions with the mean values from Table 1 and the assumed coefficient of variation of 0.2 . The corresponding failure forces for the springs can then be solved for from Eq. (28):

$$
f_{\text {fail }, j}=\frac{\left|P_{j} \phi_{1}\right|}{\omega_{1}} \sqrt{\left.2 \varepsilon_{f a i l, 1}\right|_{j}}
$$

Although these failure forces are generated to match the desired mean and standard deviation of the strain energies at failure in the first mode, they also match these quantities for the second mode. To see this, note that, by definition, the generated failure forces are such that

$$
\begin{aligned}
& \boldsymbol{E}\left[\left.\varepsilon_{f a i l, 1}\right|_{j}\right]=\frac{1}{2} \frac{\omega_{1}^{2}}{\left|P_{j} \phi_{1}\right|^{2}} \boldsymbol{E}\left[f_{\text {fail }, j}^{2}\right]=\frac{1}{2} \frac{\omega_{1}^{2}}{\left|P_{j} \phi_{1}\right|^{2}} \bar{f}_{\text {fail }, j}^{2} \\
& \boldsymbol{\sigma}\left[\left.\varepsilon_{\text {fail }, 1}\right|_{j}\right]=\frac{1}{2} \frac{\omega_{1}^{2}}{\left|P_{j} \phi_{1}\right|^{2}} \boldsymbol{\sigma}\left[f_{f a i l, j}^{2}\right]=0.2 \cdot \frac{1}{2} \frac{\omega_{1}^{2}}{\left|P_{j} \phi_{1}\right|^{2}} \bar{f}_{\text {fail }, j}^{2}
\end{aligned}
$$

Multiplying each of these equations by $\omega_{2}^{2}\left|P_{j} \phi_{1}\right|^{2} /\left(\omega_{1}^{2}\left|P_{j} \phi_{2}\right|^{2}\right)$ shows that the generated failure forces are such that the strain energies at failure in the second mode have the means given in Table 1 and coefficient of variation of 0.2 , as desired:

$$
\boldsymbol{E}\left[\left.\varepsilon_{f a i l, 2}\right|_{j}\right]=\frac{1}{2} \frac{\omega_{2}^{2}}{\left|P_{j} \phi_{2}\right|^{2}} \boldsymbol{E}\left[f_{\text {fail }, j}^{2}\right]=\frac{1}{2} \frac{\omega_{2}^{2}}{\left|P_{j} \phi_{2}\right|^{2}} \bar{f}_{\text {fail }, j}^{2}
$$


Table 4

Percentage difference between nominal values and actual values from Monte Carlo analysis for mean and standard deviation of peak modal strain energies and strain energies at failure

\begin{tabular}{|c|c|c|c|c|c|c|c|c|}
\hline & \multicolumn{2}{|c|}{ Structure 1} & \multicolumn{2}{|c|}{ Structure 2} & \multicolumn{2}{|c|}{ Structure 3} & \multicolumn{2}{|c|}{ Structure 4} \\
\hline & Mode 1 & Mode 2 & Mode 1 & Mode 2 & Mode 1 & Mode 2 & Mode 1 & Mode 2 \\
\hline$\%$ Diff. in mean of peak modal strain energies & $0.03 \%$ & $-1.88 \%$ & $0.77 \%$ & $-1.76 \%$ & $0.56 \%$ & $0.15 \%$ & $0.08 \%$ & $-1.37 \%$ \\
\hline$\%$ Diff. in Std. Dev. of peak modal strain energies & $0.59 \%$ & $-0.60 \%$ & $0.59 \%$ & $0.17 \%$ & $-0.33 \%$ & $-1.01 \%$ & $0.93 \%$ & $-1.97 \%$ \\
\hline$\%$ Diff. in mean of strain energies at failure of spring 1 & $0.09 \%$ & $0.09 \%$ & $0.02 \%$ & $0.02 \%$ & $-0.21 \%$ & $-0.21 \%$ & $0.09 \%$ & $0.09 \%$ \\
\hline$\%$ Diff. in Std. Dev. of strain energies at failure of spring 1 & $-0.69 \%$ & $-0.69 \%$ & $0.52 \%$ & $0.52 \%$ & $0.78 \%$ & $0.78 \%$ & $0.58 \%$ & $0.58 \%$ \\
\hline$\%$ Diff. in mean of strain energies at failure of spring 2 & $-0.09 \%$ & $-0.09 \%$ & $-0.05 \%$ & $-0.05 \%$ & $-0.28 \%$ & $-0.28 \%$ & $-0.23 \%$ & $-0.23 \%$ \\
\hline \% Diff. in Std. Dev. of strain energies at failure of spring 2 & $-0.35 \%$ & $-0.35 \%$ & $-0.20 \%$ & $-0.20 \%$ & $0.95 \%$ & $0.95 \%$ & $-0.78 \%$ & $-0.78 \%$ \\
\hline
\end{tabular}

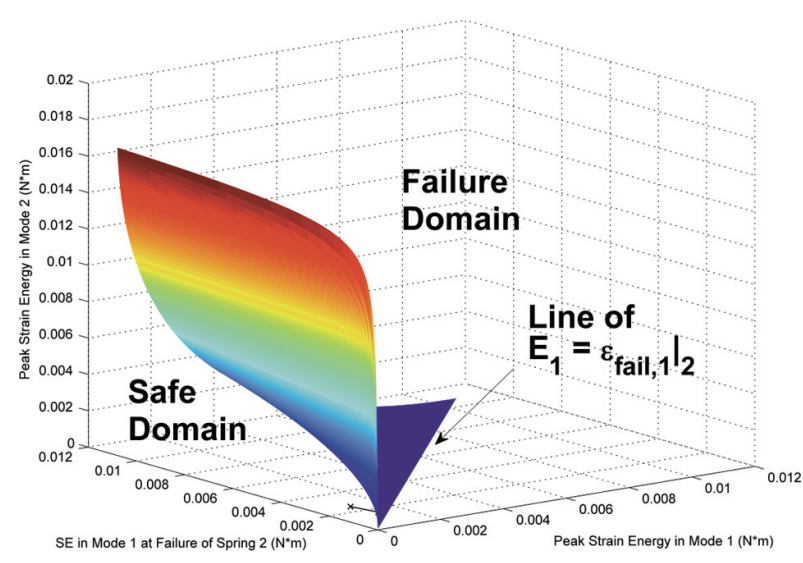

Fig. 3. Limit state surface and MPP for failure of spring 2 in structure 1 with $Z_{4}=Z_{4}^{*}$.

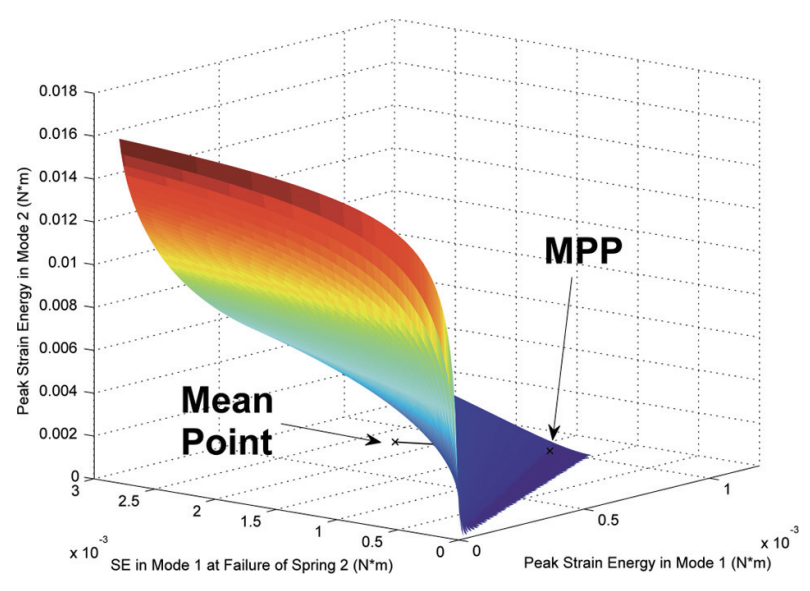

Fig. 4. Zoom-in on Fig. 3 showing MPP.

$$
\boldsymbol{\sigma}\left[\left.\varepsilon_{\text {fail }, 2}\right|_{j}\right]=\frac{1}{2} \frac{\omega_{2}^{2}}{\left|P_{j} \phi_{2}\right|^{2}} \boldsymbol{\sigma}\left[f_{f a i l, j}^{2}\right]=0.2 \cdot \frac{1}{2} \frac{\omega_{2}^{2}}{\left|P_{j} \phi_{2}\right|^{2}} \bar{f}_{f a i l, j}^{2}
$$

Once the base acceleration and failure forces have been generated as described above, the response of the structure is integrated over $300 \mathrm{~ms}$ using the central difference method. Table 2 shows the reliabilities from Monte Carlo analysis with $10^{4}$ trials for each of the four structures. Table 4 shows the percentage difference between the nominal values from Table 1 and the actual values from the Monte Carlo analyses of the means and standard deviations of the peak modal strain energies and modal strain energies at failure. Note that all of these differences are less than $2 \%$, so the base accelerations and failure forces used in the Monte Carlo analysis closely match the second-moment properties of these variables used in the reliability analysis.

The results from the Monte Carlo analyses shown in Table 2 give confidence in the ability of the reliability index for the energy-based limit state function to predict structural reliability. The results confirm the prediction that structure 1 is by far the most reliable, followed by structures 3,2 , and 4 . In addition, the results confirm that structures 1 and 3 are most likely to fail at spring 2, while structures 2 and 4 are most likely to fail at spring 1 .

In addition to illustrating the use of the energy-based limit state function in level II reliability analysis, the two DOF structures from Fig. 1 are also useful for visualizing the limit state surface. The limit state surface for each of these structures is a three-dimensional hypersurface in the four-dimensional $Z$-space. In order to visualize these surfaces, consider the limit state surface corresponding to failure of spring 2 for structure 1 . Suppose that the strain energy at failure in the second mode, $Z_{4}$, is fixed at its value at the MPP, $Z_{4}^{*}$. In this case, the limit state surface is a two-dimensional surface defined by

$$
Z_{2}=Z_{4}^{*}\left(1-\sqrt{\frac{Z_{1}}{Z_{3}}}\right)^{2} \text { for } Z_{1}<Z_{3}
$$




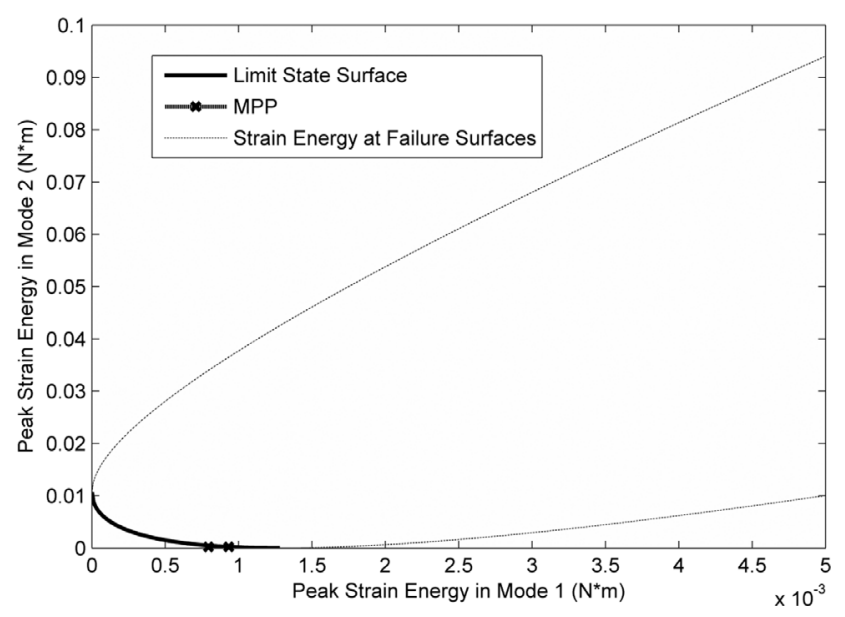

Fig. 5. Limit state surface, strain energy at failure surfaces, and MPP for failure of spring 2 in structure 1 with $Z_{3}=Z_{3}^{*}$ and $Z_{4}=Z_{4}^{*}$.

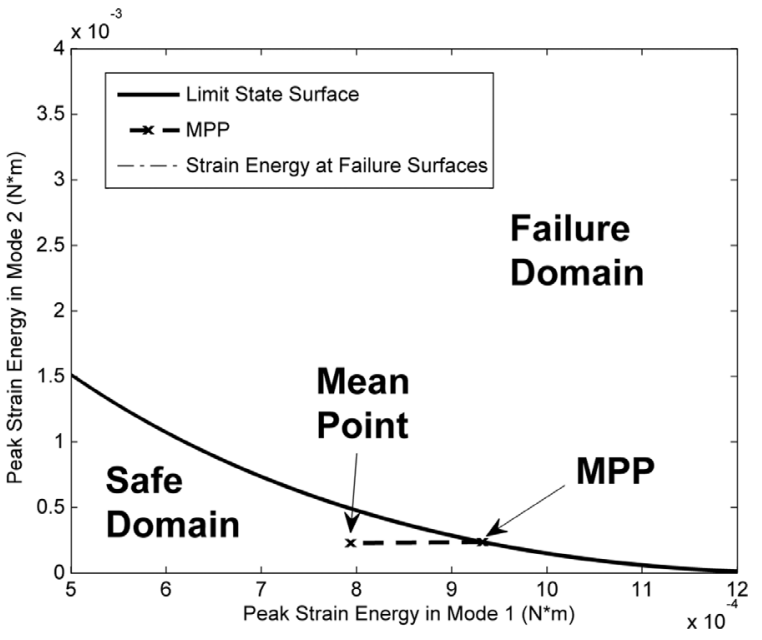

Fig. 6. Zoom-in on Fig. 5 showing MPP.

This limit state surface is plotted in Figs 3 and 4 along with the vector from the mean point to the MPP. These figures illustrate how the $Z$-space is partitioned by the limit state surface into a failure domain and a safe domain. Note that the vector from the mean point to the MPP in Figs 3 and 4 does not necessarily represent the shortest distance from the mean point to the limit state surface, as the MPP is defined as the point on the limit state surface nearest the mean point in the $X$-space. Also note that the limit state surface is undefined for values of $E_{1}$ greater than $\left.\varepsilon_{\text {fail }, 1}\right|_{2}$ since the limit state function is strictly negative in this region. Physically, this means that when the peak strain energy into mode 1 is greater than the strain energy at failure for mode 1, the structure fails regardless of the peak strain energy into mode 2 .

To gain further insight into the nature of the limit state surface, consider failure of spring 2 for structure 1 and fix both the strain energy at failure in mode 1 and the strain energy at failure in mode 2 at their values at the MPP, $Z_{3}^{*}$ and $Z_{4}^{*}$, respectively. This is equivalent to slicing the limit state surface shown in Figs 3 and 4 along the plane $Z_{3}=Z_{3}^{*}$. In this case, the limit state surface is simply a curve in a two-dimensional space and is defined by

$$
Z_{2}=Z_{4}^{*}\left(1-\sqrt{\frac{Z_{1}}{Z_{3}^{*}}}\right)^{2} \text { for } Z_{1}<Z_{3}^{*}
$$

This limit state surface is plotted in Figs 5 and 6 along with the vector from the mean point to the MPP and the strain energy at failure surfaces defined in Eq. (8). Note that, although the system can fail at any point on the strain energy at failure surfaces, the limit state surface consists of only their most conservative portion. Thus the system cannot fail without the strain energy piercing one of the limit state surfaces, but it is not guaranteed to fail in this case since it might actually fail on one of the less conservative strain energy at failure surfaces. To clarify this issue, consider the two states $x=\alpha_{1} \phi_{1}+\alpha_{2} \phi_{2}$ and $y=\alpha_{1} \phi_{1}-\alpha_{2} \phi_{2}$, which have the same modal strain energies, but different strain energies at failure since they produce different spring forces. Thus for a given strain energy into the first mode, there are two distinct values of strain energy in the second mode which will cause the system to fail, depending upon the relative phase of the modes. In adopting the most conservative strain energy at failure surface as the limit state surface, it is assumed that the worst-case phasing of the modes occurs at all times. It is expected that this assumption will introduce considerable conservative bias, but as discussed earlier, this conservative bias might not be important when the limit state function is used for level II reliability analysis, since it should affect the reliability indices for various structures equally. For example, although the reliability indices presented in Table 2 are probably quite conservative, they should all be roughly equally conservative, so the conclusion that structure 1 is by far the most reliable in the given environment still holds.

The somewhat counterintuitive nature of the positive branch $\left(s_{1}=1 \mathrm{in} \mathrm{Eq.} \mathrm{(8))} \mathrm{of} \mathrm{the} \mathrm{strain} \mathrm{energy} \mathrm{at} \mathrm{failure}\right.$ surface and the negative branch for $\varepsilon_{1}>\left.\varepsilon_{f a i l, 1}\right|_{j}$, for which the peak strain energy in the second mode required to fail 
Table 5

Nominal material properties used in finite element model

\begin{tabular}{lcccc}
\hline & Elastic modulus $(\mathrm{GPa})$ & Density $\left(\mathrm{kg} / \mathrm{m}^{3}\right)$ & Poisson's ratio & Yield stress $(\mathrm{MPa})$ \\
\hline Steel & 200 & 7861 & 0.30 & 552 \\
Aluminum & 68.9 & 2700 & 0.33 & 276 \\
\hline
\end{tabular}

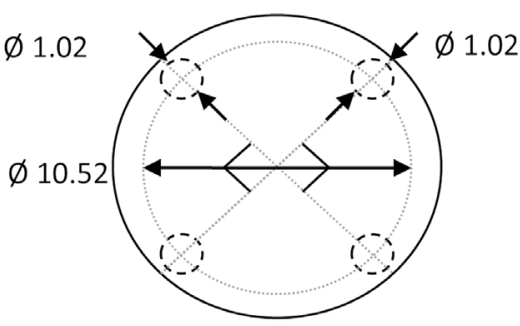

Varied from 0.1588 to 1.27

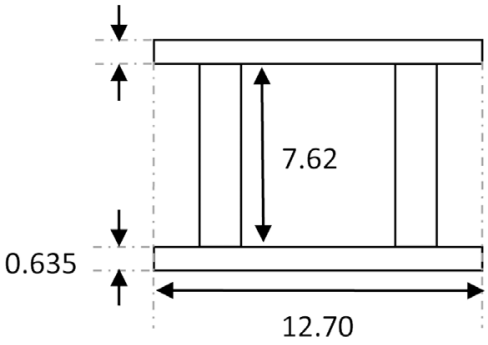

Fig. 7. Drawing of a simple structure consisting of two circular plates joined by four legs (dimensions in $\mathrm{cm}$ ).

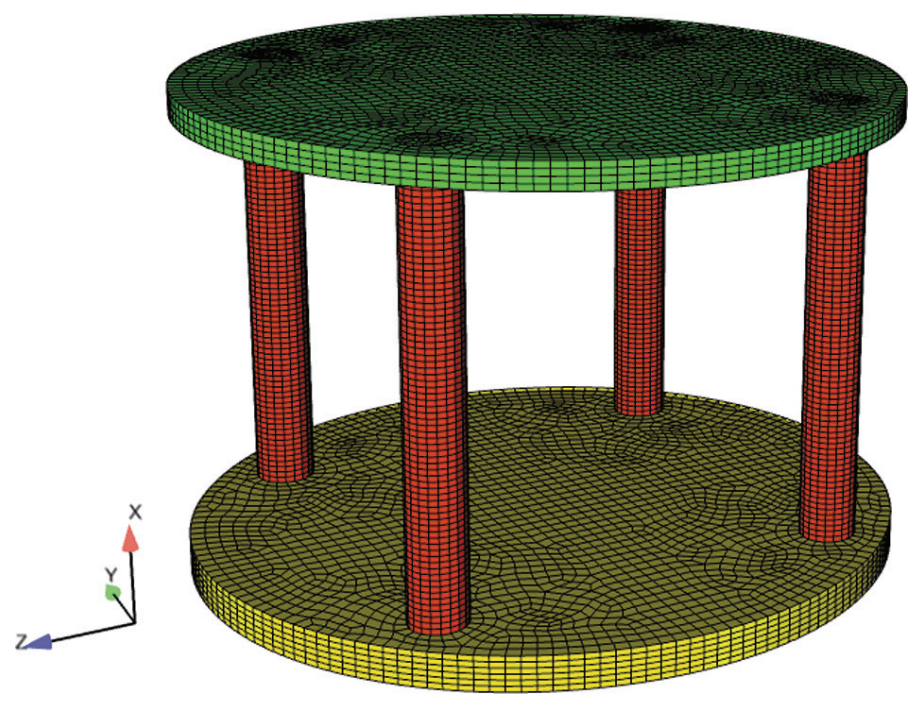

Fig. 8. Finite element model of structure from Fig. 7.

the structure increases as the peak strain energy in the first mode increases, is the result of a singularity in which the modal displacements produce vanishing force in one of the springs. For example, consider the shape $x=\alpha_{1} \phi_{1}+\phi_{2}$ for varying values of $\alpha_{1}$ and denote the value of $\alpha_{1}$ for which the force in the first spring vanishes by $\tilde{\alpha}_{1}$. Then as $\alpha_{1} \rightarrow \tilde{\alpha}_{1}$, the strain energy at failure of the first spring in both modes approaches infinity since the force in the first spring is vanishing and the scale factor to failure is approaching infinity. Since these branches of the strain energy at failure surface are not included in the definition of the limit state function, they are not considered further in this paper.

\section{Application to a simple finite element model}

A more realistic application of the energy-based reliability approach is provided by the structure shown in Fig. 7. This structure consists of two circular plates joined together by four legs with circular cross sections. The base plate is composed of steel while the legs and top plate are composed of aluminum. The nominal properties used for these materials are listed in Table 5. A finite element model of this structure consisting of approximately 36,000 8-node hexahedral elements was constructed and is shown in Fig. 8.

The structure is subjected to the nominal base acceleration shown in Fig. 9 and is considered to have failed if the Von Mises stress in any element exceeds the corresponding yield stress. For brevity, only axial (x-direction) shocks are considered here, but the same approach could be used to analyze transverse shocks if desired. Secondmoment information for the environmental severity and structural failure characteristics, described here by the peak modal strain energies and modal strain energies at failure, is assumed to be available and the thickness of the top plate is to be selected in order to maximize the reliability of the structure in the given environment. This problem, although greatly simplified, is representative of many problems frequently encountered in the design of structures for shock environments. In many cases, such problems are addressed simply by analyzing each design for the nominal case and determining the safety factor. While this approach can provide quick design guidance, it fails to account 
Table 6

Natural frequencies, modal participation factors, and nominal peak modal strain energies for structure from Fig. 7

\begin{tabular}{|c|c|c|c|c|c|c|c|}
\hline Mode \# & $f(\mathrm{~Hz})$ & $\mathrm{MPF}-x$ & $E(\mathrm{~N} \cdot \mathrm{m})$ & Mode \# & $f(\mathrm{~Hz})$ & $\mathrm{MPF}-x$ & $E(\mathrm{~N} \cdot \mathrm{m})$ \\
\hline \multicolumn{4}{|c|}{$0.3175 \mathrm{~cm}$ thickness } & \multicolumn{4}{|c|}{$0.4763 \mathrm{~cm}$ thickness } \\
\hline 4 & 1974 & -0.282 & 0.671 & 5 & 2397 & 0.442 & 1.323 \\
\hline 8 & 2638 & -0.636 & 1.613 & 6 & 2735 & 0.701 & 2.392 \\
\hline 9 & 2850 & -0.418 & 0.712 & 9 & 3876 & 0.082 & 0.0208 \\
\hline 18 & 5314 & -0.074 & 0.016 & 16 & 5844 & 0.017 & 0.00086 \\
\hline 27 & 8262 & 0.103 & 0.019 & 26 & 9944 & 0.310 & 0.136 \\
\hline 30 & 9956 & -0.303 & 0.119 & 33 & 11789 & 0.118 & 0.0028 \\
\hline 37 & 11993 & -0.227 & 0.0088 & 34 & 12375 & 0.179 & 0.0050 \\
\hline 44 & 14371 & -0.105 & 0.00083 & 45 & 15643 & -0.078 & 0.00036 \\
\hline \multicolumn{4}{|c|}{$0.635 \mathrm{~cm}$ thickness } & \multicolumn{4}{|c|}{$1.27 \mathrm{~cm}$ thickness } \\
\hline 5 & 2611 & 0.799 & 2.927 & 4 & 2539 & -0.878 & 5.175 \\
\hline 6 & 2854 & 0.260 & 0.314 & 7 & 3458 & -0.144 & 0.114 \\
\hline 9 & 4729 & 0.132 & 0.059 & 12 & 6135 & -0.030 & 0.0041 \\
\hline 16 & 6220 & -0.015 & 0.00076 & 20 & 7704 & -0.099 & 0.0292 \\
\hline 27 & 10434 & -0.303 & 0.062 & 25 & 11855 & -0.272 & 0.0190 \\
\hline 29 & 12411 & 0.166 & 0.0045 & 29 & 14545 & 0.039 & 0.00015 \\
\hline 34 & 14150 & 0.072 & 0.00047 & 38 & 16880 & 0.050 & 0.00016 \\
\hline 44 & 16824 & -0.051 & 0.00013 & - & - & - & - \\
\hline
\end{tabular}

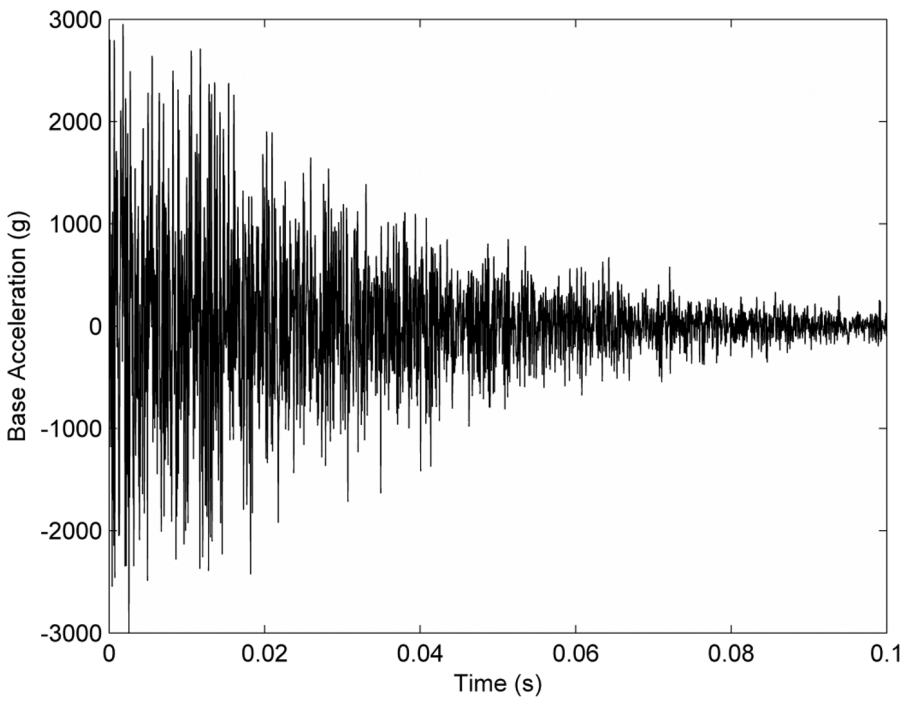

Fig. 9. Nominal base acceleration applied to structure from Fig. 7.

for uncertainties in both the environment and structure and can therefore be inaccurate. However, the alternative approach is Monte Carlo analysis, which can often be too computationally intensive to provide the quick turnaround necessary to support the design process. As demonstrated below, a level II reliability analysis using the energy-based limit state function can allow for quick turnaround while still accounting for relevant uncertainties.

Top plate thicknesses ranging from 0.159 to $1.35 \mathrm{~cm}$ were considered. For each of 43 uniformly spaced thicknesses in this range, a modal analysis was conducted in which the outer rim of the base plate's bottom surface was fixed. The first 50 natural frequencies, $\mathrm{x}$-direction modal participation factors, and nominal peak modal strain energies (Eq. (31)) were computed for each thickness. Table 6 shows these quantities for four of the thicknesses considered. Only modes with nominal peak strain energy greater than $10^{-5}$ times the maximum peak modal strain energy are included in Table 6. The nominal strain energy at failure of element $j$ in mode $i$ is given by

$$
\left.\varepsilon_{f a i l, i}\right|_{j}=\frac{1}{2} \omega_{i}^{2} \frac{\bar{\sigma}_{Y, j}^{2}}{\sigma_{V M, i j}^{2}}
$$


Table 7

Sensitivity vectors for structure of Fig. 7

\begin{tabular}{lrlrlrlr}
\hline $0.3175 \mathrm{~cm}$ thickness & \multicolumn{2}{l}{$0.4763 \mathrm{~cm}$ thickness } & \multicolumn{2}{l}{$0.635 \mathrm{~cm}$ thickness } & \multicolumn{2}{c}{$1.27 \mathrm{~cm}$ thickness } \\
\hline $\mathrm{E}_{4}$ & -0.158 & $\mathrm{E}_{5}$ & -0.090 & $\mathrm{E}_{5}$ & -0.464 & $\mathrm{E}_{4}$ & -0.576 \\
$\mathrm{E}_{8}$ & -0.175 & $\mathrm{E}_{6}$ & -0.035 & $\mathrm{E}_{6}$ & -0.094 & $\mathrm{E}_{7}$ & -0.036 \\
$\mathrm{E}_{9}$ & -0.109 & $\mathrm{E}_{9}$ & -0.008 & $\mathrm{E}_{9}$ & -0.034 & $\mathrm{E}_{20}$ & -0.015 \\
$\mathrm{E}_{30}$ & -0.013 & $\mathrm{E}_{26}$ & -0.020 & $\mathrm{E}_{27}$ & -0.030 & $\mathrm{E}_{25}$ & -0.015 \\
$\varepsilon_{\text {fail }, 4}$ & 0.315 & $\varepsilon_{\text {fail }, 5}$ & 0.559 & $\varepsilon_{\text {fail }, 5}$ & 0.644 & $\varepsilon_{\text {fail }, 4}$ & 0.735 \\
$\varepsilon_{\text {fail }, 8}$ & 0.348 & $\varepsilon_{\text {fail }, 6}$ & 0.211 & $\varepsilon_{\text {fail }, 6}$ & 0.120 & $\varepsilon_{\text {fail }, 7}$ & 0.042 \\
$\varepsilon_{\text {fail }, 9}$ & 0.217 & $\varepsilon_{\text {fail }, 9}$ & 0.049 & $\varepsilon_{\text {fail }, 9}$ & 0.043 & $\varepsilon_{\text {fail }, 20}$ & 0.018 \\
$\varepsilon_{\text {fail }, 30}$ & 0.026 & $\varepsilon_{\text {fail }, 26}$ & 0.122 & $\varepsilon_{\text {fail }, 27}$ & 0.037 & $\varepsilon_{\text {fail }, 25}$ & 0.018 \\
\hline \multicolumn{7}{r}{} & \multicolumn{7}{c}{}
\end{tabular}

where $\bar{\sigma}_{Y, j}$ is the nominal yield stress for element $j$ and $\sigma_{V M, i j}$ is the Von Mises stress in element $j$ for massnormalized mode $i$. Note that, because Von Mises stress is not a linear function of displacement, it does not satisfy the assumptions underlying the limit state function. Nonetheless, it is still used to define failure for this structure and the resulting reliability indices agree well with Monte Carlo analysis, so it appears that this inconsistency does not have a large effect on the accuracy. Future work will examine the error introduced by this assumption more quantitatively.

For each of the elements, the vector of basic variables is given in Eq. (16) and the limit state function and its gradient are given in Eqs (17) and (24). For each candidate thickness and each element, the expected value of $Z$ can be evaluated using Eqs (31) and (47). Each basic variable is assigned a coefficient of variation equal to 0.5 except for the modal strain energies at failure for the elements in the top plate, which are given coefficients of variation equal to 1.0. This corresponds to a situation in which there is greater uncertainty in the yield stress of the top plate than in that of the other components, as might be the case if the top plate is produced by a different supplier. Given the assumptions about the correlation between the basic variables discussed earlier, the covariance matrix for $Z$ can be evaluated. The iterative procedure given in Eq. (23) was applied to find the MPP, and hence the Hasofer-Lind reliability index, for each of the candidate thicknesses. The reliability index, along with the reliability indices for the top plate, base plate, and legs, is plotted as a function of top plate thickness in Fig. 10. The sensitivity vector from Eq. (27) is given in Table 7 for four values of the top plate thickness. To ensure that the 50 modes included in the computation were sufficient to obtain converged values of the reliability index, the number of modes shapes was increased to 100 for thicknesses of $0.3175,0.4763,0.635$, and $1.27 \mathrm{~cm}$. In these four cases, the reliability index changed by a maximum of $6.6 \%$, which was deemed to be a sufficient level of convergence. In addition to doubling the number of mode shapes, standard rules of thumb such as including modes with natural frequencies up to twice the maximum frequency of the input can be used to determine an appropriate number of modes. For comparison with the reliability indices, a modal transient analysis of the nominal case (i.e., yield stresses from Table 5 and base acceleration from Fig. 9) was performed for each candidate thickness and the safety factor was calculated as

$$
S F=\min _{i} \frac{\bar{\sigma}_{Y, i}}{\hat{\sigma}_{V M, i}}
$$

where $i$ ranges from 1 to the number of elements and $\hat{\sigma}_{V M, i}$ represents the peak Von Mises stress in element $i$ over all time steps. The safety factors computed in this way are plotted as a function of top plate thickness in Fig. 11, along with the safety factors for the top plate, base plate, and legs. In the modal transient analyses, the first 50 modes were retained, a timestep of $10^{-6} \mathrm{~s}$ was used, the base acceleration from Fig. 9 was enforced along the outer rim of the bottom surface of the base plate, and the response was integrated over $300 \mathrm{~ms}$.

The reliability indices from Fig. 10 suggest that increasing the thickness of the top plate from $0.159 \mathrm{~cm}$ to $0.82 \mathrm{~cm}$ generally increases the reliability of the structure, although there are a few local minima in the reliability index around $0.3,0.4$, and $0.6 \mathrm{~cm}$. For thicknesses from 0.159 to $0.82 \mathrm{~cm}$, the minimum reliability index is associated with the top plate, except for thicknesses between 0.50 and $0.71 \mathrm{~cm}$, for which the minimum reliability index is associated with the legs. As the thickness is increased beyond $0.82 \mathrm{~cm}$, the minimum reliability index is associated with the base plate and the reliability is reduced for further increases in the thickness. The reliability index is maximized at a top plate thickness of $0.82 \mathrm{~cm}$. By comparison, the safety factors from Fig. 11 are maximized at a thickness of $0.37 \mathrm{~cm}$. The discrepancy between the safety factors and the reliability indices is a result of the safety factor not accounting for variation in both the structure and the environment. 


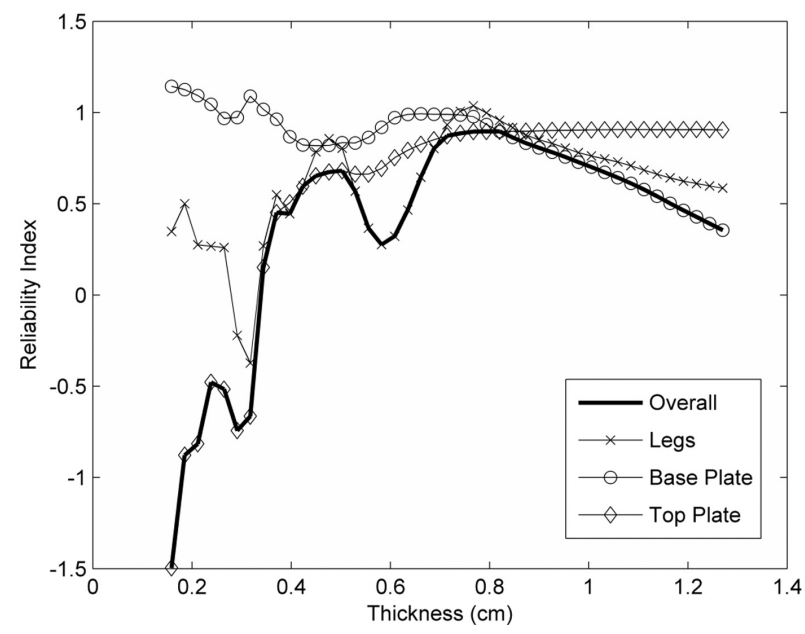

Fig. 10. Reliability indices vs. top plate thickness for structure from Fig. 7.

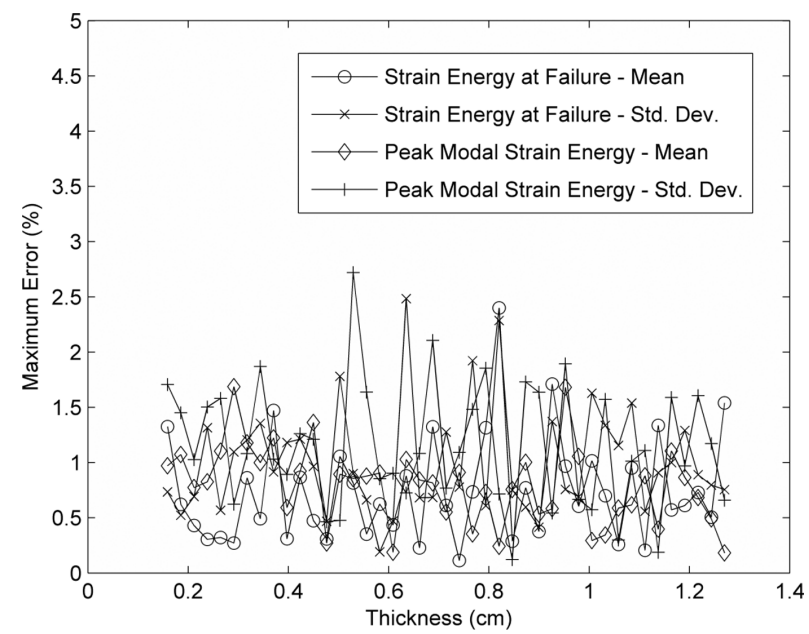

Fig. 12. Percentage difference between nominal values and actual values from Monte Carlo analysis of means and standard deviations of peak modal strain energies and modal strain energies at failure.

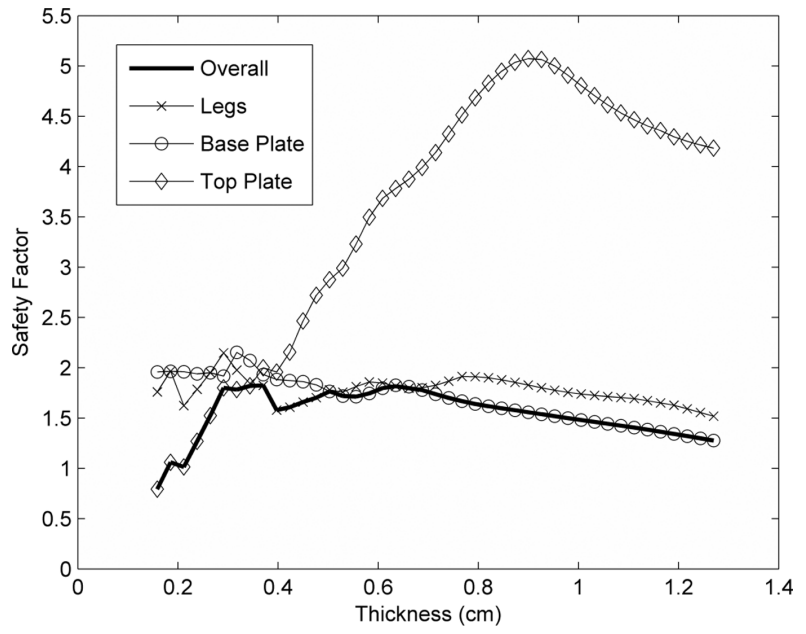

Fig. 11. Safety factors vs. top plate thickness for structure from Fig. 7.

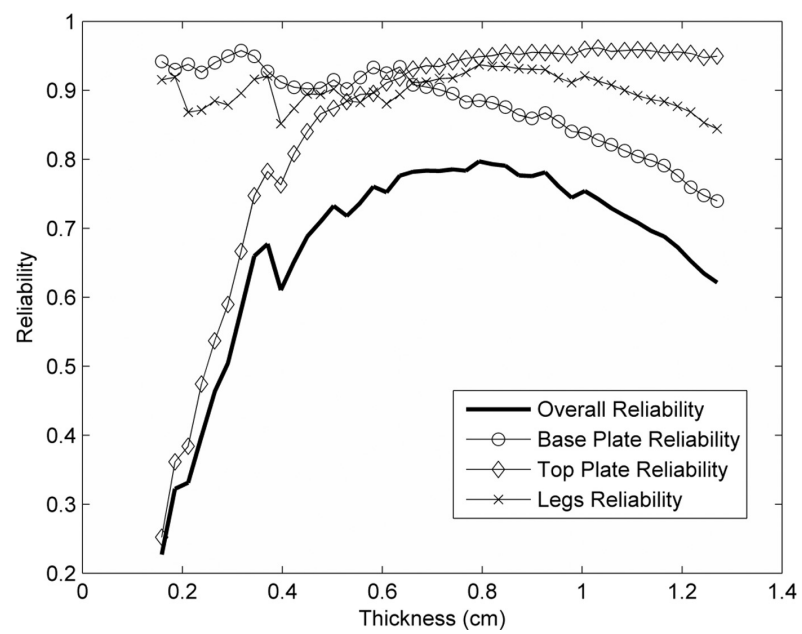

Fig. 13. Reliability from Monte Carlo analysis for structure from Fig. 7.

In addition to the reliability index, the sensitivity vectors listed in Table 7 also provide information that could be useful in the design process. By identifying the modes which affect the reliability most strongly, they suggest design changes that could make the structure more reliable, such as altering the design to shift the frequencies of key modes so that they receive less energy or to increase the strain energy at failure for key modes. For example, the sensitivity vectors show that, for a top plate thickness of $0.4763 \mathrm{~cm}$, the structure is most sensitive to the peak strain energy and strain energy at failure in mode 5, in spite of the nominal peak strain energy in mode 5 being only about half of that in mode 6.

In order to provide confidence in the results from the reliability analysis, a Monte Carlo analysis was also performed. Just as for the two DOF systems considered earlier, base accelerations were generated as exponentially decayed sums of sines at a subset of the structure's natural frequencies:

$$
A_{g}=e^{-c t} \sum_{i \in \mathrm{K}} A_{i} \sin \omega_{i} t
$$




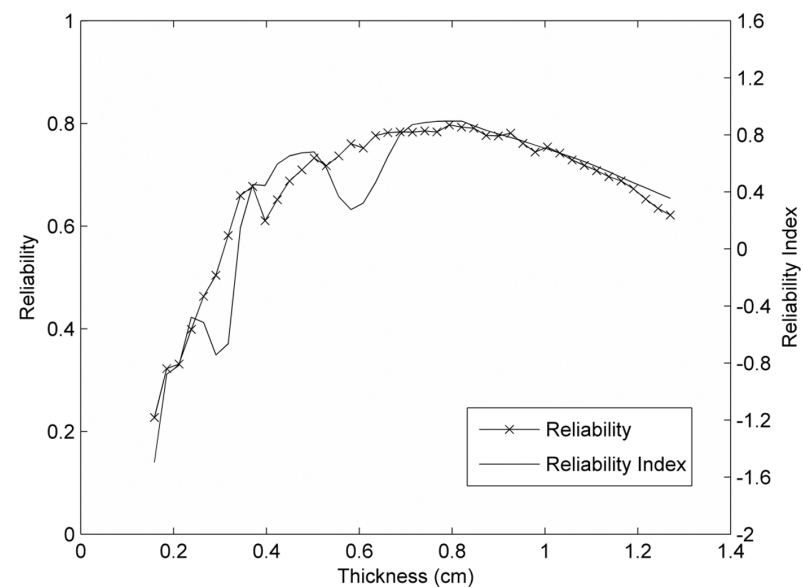

Fig. 14. Overlay of reliability index and Monte Carlo reliability vs. top plate thickness.

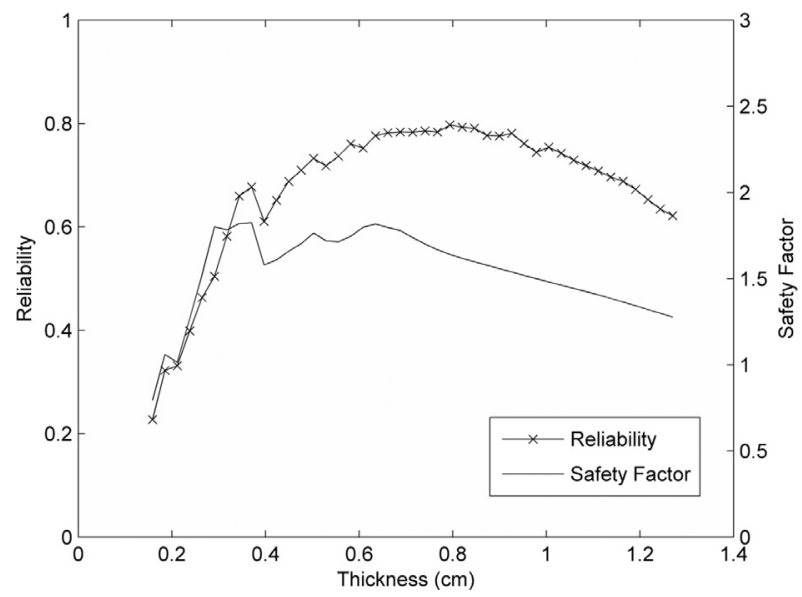

Fig. 15. Overlay of safety factor and Monte Carlo reliability vs. top plate thickness.

where $K$ denotes the indices of the selected modes and the decay constant was chosen to be $c=151 / \mathrm{s}$. Only those modes with nominal peak strain energy greater than $1 \%$ of the maximum peak modal strain energy were included in set $K$. These modes are listed in bold in Table 6 for four of the thicknesses considered. The coefficients $A_{i}$ are independent and normally distributed with means and variances selected using the approach discussed in Section 4. On each Monte Carlo trial, the strain energy at failure of the first element in the top plate in the first mode is drawn from a Weibull distribution with the mean value from Eq. (47) and the assumed coefficient of variation of 1.0. The corresponding yield stress for this element can then be solved for from Eq. (47):

$$
\sigma_{Y, j}=\frac{\sigma_{V M, 1 j}}{\omega_{1}} \sqrt{\left.2 \varepsilon_{f a i l, 1}\right|_{j}}
$$

This value is then taken to be the yield stress for all of the elements in the top plate and the procedure is repeated to generate yield stresses for the base plate and legs. Although these yield stresses are generated to match the desired mean and standard deviation of the strain energies at failure in the first mode, they can be shown to match these quantities for all modes using the approach taken in Section 4. Once the base acceleration and yield stresses have been generated, the response of the structure is integrated over $30 \mathrm{~ms}$ in a modal transient analysis with the first 50 modes retained. If the Von Mises stress in any element exceeds the corresponding yield stress, a failure is recorded. Figure 12 shows the percentage difference between the nominal values and the actual values from the Monte Carlo analyses of the means and standard deviations of the peak modal strain energies and modal strain energies at failure. These errors are all less than 3\%, so the second moment properties of the base accelerations and yield stresses used in the Monte Carlo analyses are considered to accurately reflect the assumptions made in the reliability analysis.

Figure 13 shows the failure rates from Monte Carlo analysis with $10^{4}$ trials for each thickness. The failure rates show the same trend as the reliability indices: the reliability increases with the thickness of the top plate up to a value of $0.79 \mathrm{~cm}$ and then decreases with further increases in the thickness. For thicknesses between $0.159 \mathrm{~cm}$ and $0.53 \mathrm{~cm}$, the reliability is governed by the top plate and, for thicknesses $0.53 \mathrm{~cm}$ and $0.66 \mathrm{~cm}$, by the legs. For thicknesses beyond $0.66 \mathrm{~cm}$, the reliability is governed by the base plate. This is qualitatively similar to the trend suggested by the reliability analysis, with the only major difference being that the local minima in the reliability of the legs around 0.3 and $0.6 \mathrm{~cm}$ are exaggerated by the reliability index.

The reliability from the Monte Carlo analysis is compared directly with the reliability index in Fig. 14 and the safety factor in Fig. 15. These figures show that the reliability index provides a means of comparing and controlling reliability which is qualitatively consistent with the results from the Monte Carlo analysis, while the safety factor does not, due to its inability to account for uncertainties. It was possible to do Monte Carlo analysis in a relatively timely fashion for this problem (approximately 30 hours on a single processor for $10^{4}$ trials) because the model is quite simple and because the reliability is very low, so a fairly small number of trials was required. In practical structural reliability problems, the model will typically be more complex and the failure rate will be much lower, 
perhaps on the order of one-in-a-million, so a much larger number of trials will be required. While Monte Carlo analysis might still be possible in some of these cases, it would require substantial computational resources and time. In situations where quick, qualitative comparison of several designs is required, such an approach would be too slow and inflexible. The use of level II reliability analysis with the energy-based limit state function provides an alternative approach which requires only a modal analysis and some simple post-processing of the results, but still accounts for relevant uncertainties in the structure and environment. In addition, it provides valuable information about the spectral sensitivity of the reliability.

\section{Conclusion}

A limit state function based upon the peak modal strain energies and modal strain energies at failure has been developed for the estimation of structural reliability in shock environments. The Hasofer-Lind reliability index was briefly reviewed and it was shown how this index can be computed for the energy-based limit state function. Level II reliability analysis using the energy-based limit state function was performed for two DOF mass-spring systems and a simple finite element model and, in both cases, the results were seen to agree well with those from Monte Carlo analysis.

The reliability index for the energy-based limit state function provides an accurate and easily calculated measure of a structure's reliability. Use of this index could be very valuable during the design process, when fast, qualitative comparison of the relative reliabilities of various design options is required. In such situations, Monte Carlo analysis is often too computationally intensive to provide the fast turnaround required to support design studies. The reliability approach developed here requires little effort beyond a modal analysis, but still accounts for relevant uncertainties in both the structure and environment. In addition, the reliability approach developed here provides important information about the sensitivity of the structure's reliability to the frequency content of the input.

\section{Acknowledgments}

Sandia National Laboratories is a multi-program laboratory managed and operated by Sandia Corporation, a wholly owned subsidiary of Lockheed Martin Corporation, for the U.S. Department of Energy's National Nuclear Security Administration under contract DE-AC04-94AL85000. The author would also like to acknowledge the important contributions of Tim Edwards to this work, both in developing the energy-based characterization of shock and vibration environments and in encouraging its extension to the prediction of structural reliability.

\section{References}

[1] D.E. Hudson, Response spectrum techniques in engineering seismology, Proceedings of the World Conference on Earthquake Engineering, Berkeley, California, (1956), 4-1, 4-12.

[2] G.W. Housner, Behavior of structures during earthquake, Journal of the Engineering Mechanics Division (ASCE) 85(4) (1959), 109-129.

[3] G.W. Housner, Limit design of structures to resist earthquakes, Proceedings of the World Conference on Earthquake Engineering, Berkeley, California, (1956), 5-1, 5-13.

[4] T.S. Edwards, Power delivered to mechanical systems by random vibrations, Shock and Vibration 16(3) (2009), $261-271$.

[5] T.S. Edwards, Using work and energy to characterize mechanical shock, Technical Report SAND2007-0851J, Sandia National Laboratories, Albuquerque, NM, February 2007.

[6] T.S. Edwards, D.O. Smallwood and D.J. Segalman, Modal deposition of shock energy, Technical Report SAND2007-0691J, Sandia National Laboratories, Albuquerque, NM, February 2007.

[7] H.O. Madsen, S. Krenk and N.C. Lind, Methods of structural safety, Dover Publications, Mineola, NY, 1986.

[8] P. Thoft-Christensen and M.J. Baker, Structural reliability theory and its applications, Springer-Verlag, Berlin, 1982.

[9] A.M. Hasofer and N.C. Lind, Exact and invariant second-moment code format, Journal of the Engineering Mechanics Division (ASCE) 100 (1974), 111-121.

[10] C.A. Cornell, A probability-based structural code, Journal of the American Concrete Institute 66(12) (1969), 974-985. 

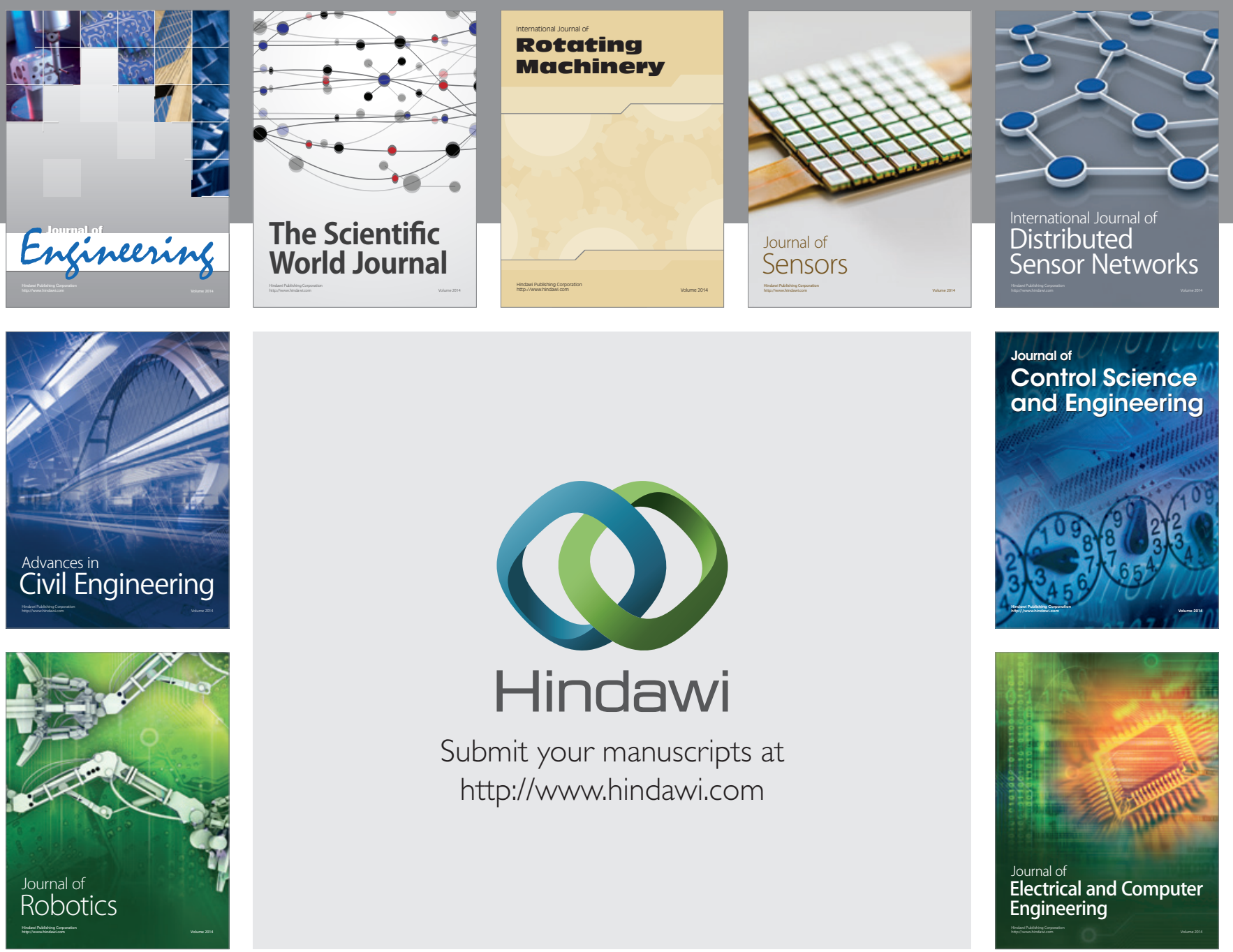

Submit your manuscripts at

http://www.hindawi.com
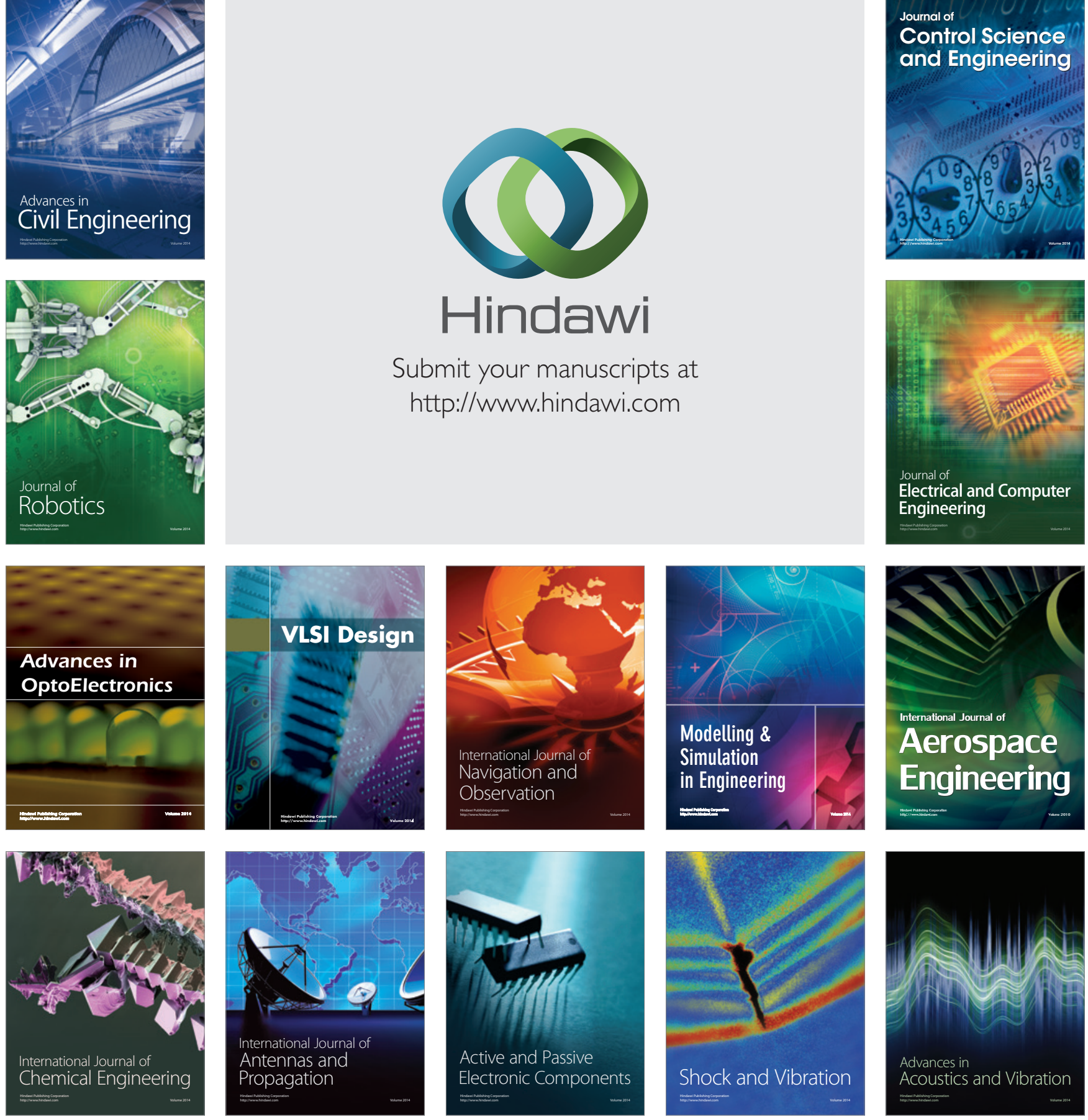